59 TRANSLATIONAL FRAMEWORK FOR REGULATORY RESEARCH THROUGH CRITICAL APPRAISAL AND EVALUATION OF POST-AUTHORIZATION SAFETY AND EFFICACY STUDIES (TRACE-PASS/PAES)

${ }^{1}$ Christof Prugger, ${ }^{2}$ Angela Spelsberg, ${ }^{3}$ Ulrich Keil. ${ }^{1}$ Institute of Public Health, Charité Universitätsmedizin Berlin, Berlin, Germany; ${ }^{2}$ Comprehensive Cancer Centre Aachen, Aachen, Germany; ${ }^{3}$ Institute of Epidemiology and Social Medicine, University of Münster, Münster, Germany

\subsection{6/bmjebm-2019-EBMLive.67}

Objectives Spontaneous reporting schemes show an increase in adverse drug events, while at the same time accelerated and conditional approval procedures for early marketing of drugs were intensified over recent years, illustrating the need to strengthen drug safety and efficacy evaluation in post-authorization studies (PAS). The European Medicines Agency (EMA) mandates and supervises post-authorization safety and efficacy studies (PASS/PAES) on approved drugs. However, unpublished study documents and results so far hamper the systematic scientific evaluation of these PAS. Developing a translational framework for the evaluation of PAS thus is an important challenge in regulatory research. The project's objective is to develop a TRAnslational framework for regulatory research through Critical appraisal and Evaluation of Post-Authorisation Safety and Efficacy Studies (TRACE-PASS/PAES).

Method TRACE-PASS/PAES will set up a database of PAS that have been registered in Germany since 2010, and it will follow up these PAS identifying study protocols and reports in public databases and obtaining access to unpublished reports using freedom of information (FOI) requests. Based on these documents from multiple data sources, the project will systematically evaluate: 1) the consistency of study set-up as described in study protocols and registration databases, 2) the transparency and consistency of reporting in journal articles and registration databases, 3) the transparency and consistency of conduct in documents held by EMA, and 4) the transparency and consistency of progress through examination of all available documents. Concerning these four aspects, the project will critically appraise documents using established, adapted, and bespoke study instruments and statistical analyses. Further, it will apply an open peer review process and open data access evaluating the four aspects across all documents.

Results We recently investigated PAS registered from 2008 to 2010 in Germany (Spelsberg et al. BMJ 2017; 356:j337). This study was the first to examine PAS using unpublished data obtained through FOI requests. Systematic evaluation of -set-up, reporting, conduct, and progress was beyond its scope. However, the study examined PASS 1160.84 on dabigatran etexilate (Pradaxa ${ }^{\circledR}$ ) using multiple data sources and showed inconsistent and non-transparent aspects in study set-up, reporting, and progress, potentially affecting the scientific quality and validity of PASS 1160.84. Detection of these inconsistencies and lack of transparency through evaluation of different data sources relating to PASS 1160.84 represents a proof of concept for the proposed project.

Conclusions Follow-up of PAS through multiple data sources proved successful in identifying shortcomings with regard to methods, reporting, and progress of a particular PAS. This concept represents an expedient strategy for the translational framework proposed by TRACE-PASS/PAES. We thus aim to extend this systematic evaluation of PAS using multiple data sources to larger samples of PAS conducted in Germany and Europe with the aim of fostering regulatory research for improved evidence and health care.

\section{METHODOLOGICAL ISSUES IN REHABILITATION RESEARCH: A SCOPING REVIEW}

${ }^{1}$ Chiara Arienti, ${ }^{2,3}$ Susan Armijo-Olivo, ${ }^{4}$ Silvia Minozzi, ${ }^{1}$ Stefano Giuseppe Lazzarini, ${ }^{1}$ Michele Patrini, ${ }^{5}$ Stefano Negrini. 'IRCCS Fondazione Don Carlo Gnocchi, Milan, Italy; ${ }^{2}$ Institute of Health Economics, Edmonton, Canada; ${ }^{3} 2$ Research Centre, Faculty of Rehabilitation Medicine, University of Alberta, Edmonton, Canada; ${ }^{4}$ Method Editor and Quality Advisor, Cochrane Review Group on Drugs and Alcohol. Department of Epidemiology, Lazio Regional Health Service, Rome, Italy; ${ }^{5}$ Department of Clinical and Experimental Sciences, University of Brescia, Brescia, Italy

\subsection{6/bmjebm-2019-EBMLive.68}

Objectives Rehabilitation interventions are usually complex and include different aspects that make difficult to accomplish with classical measures of methodological quality in clinical research. The aim of the present study was to identify, synthesize and categorize the main methodological issues in rehabilitation research to guide the development of methods for reporting and evaluating evidence in the rehabilitation field.

Method A scoping review was conducted on PubMed, Cochrane Library, EMBASE, Web of Science, Scopus, Pedro and Google Scholar database up to August 2018. Methodological studies, special communications and literature reviews addressing any methodological issue in rehabilitation research were included. For each study, we identified the methodological issues addressed. Quantitative (frequencies of issues addressed in the studies) and qualitative (content analysis of the issues) synthesis have been conducted.

Results After removing duplicates, we screened 2,879 citations, and 71 studies were finally included. Of these, 69\% $(n=49)$ were narrative reviews, $14 \%(\mathrm{n}=11)$ systematic reviews, $7 \%$ $(\mathrm{n}=5)$ editorials, 4\% $(\mathrm{n}=3)$ meta-epidemiological studies, $3 \%(\mathrm{n}=2)$ cross-sectional survey, 1\% ( $\mathrm{n}=1)$ mapping review and $1 \%(\mathrm{n}=1)$ overview. The methodological problems in rehabilitation research included: poor data collection description and statistical analysis methods (56\%); problematic application of randomized-controlled trials (38\%); interventions description (35\%); the definition of a core outcome sets for different clinical problems (31\%); lack of blinding assessor (17\%); clinical practice applicability (11\%); randomization method description (10\%); participants characteristics description and recruitment (8\%); methodological and reporting quality $(10 \%$ vs $8 \%)$. Studies also discussed other issues more related to the peer-review process, such as the methodology training need (7\%), low-quality of the peer-review process $(6 \%)$, funding $(6 \%)$ and ethical statement $(3 \%)$, lack of protocol registration (3\%), and conflict of interest declaration (1\%). Conclusions This study highlights several methodological and reporting issues in rehabilitation research and in the peer review process. Research looking at methods to improve reporting as well as improving the conduct of trials in the rehabilitation field is needed. The first step moving forward would be to evaluate the influence of all these issues on the validity of trial results and the development of a specific check-list for the evaluation of rehabilitation research specifically. 